\title{
On the Validity of Scaling Transient Conjugate Heat Transfer Characteristics
}

\author{
R. Maffulli* \\ Department of Engineering Science \\ University of Oxford \\ Oxford, OX1 3PJ, UK \\ Email: roberto.maffulli@balliol.ox.ac.uk
}

\author{
G. Marinescu \\ Retired \\ Formerly GE Power \\ Baden 5400, Switzerland
}

\author{
L. He \\ Department of Engineering Science \\ University of Oxford \\ Oxford, OX1 3PJ, UK \\ Email: li.he@eng.ox.ac.uk
}

\begin{abstract}
Accurate prediction of unsteady thermal loads is of paramount importance in several engineering disciplines and applications. Performing time-accurate unsteady Conjugate Heat Transfer (CHT) simulations presents considerable challenges due to the markedly different time scales between the solid and fluid domains. Two methods have been recently proposed, aimed at addressing this issue: MultiScale Modelling (MSM) and Equalized Time-scales (ET). The former is based on the separation of the disparate short and long temporal scales of the solution and subsequent averaging of the flow/energy equations. In the latter the equalization of the time scales is achieved through manipulation of the solid's thermal properties. Both methods are very appealing due to the possibility of being easily implemented on an existing solver. It becomes, thus, relevant to assess their performance and/or limitations. This paper work presents a comparative study of the two methods for the prediction of transient thermal load, first using a simplified case of a solid body with uniform temperature, then through the investigation of the pre-warming phase of a steam turbine. Both methods are then compared against a reference baseline fully-coupled CHT solution. The results show how the MSM allows greater accuracy and robustness with considerable saving in computational cost with respect to the baseline solution.
\end{abstract}

\section{Nomenclature \\ CHT Conjugate Heat Transfer \\ ET Equalized Time-scale \\ FC Fully Coupled \\ HTC Heat Transfer Coefficient}

\footnotetext{
*Address all correspondence related to ASME style format and figures to this author.
}

IP Intermediate Pressure

LES Large Eddy Simulation

MSM Multi Scale Modelling

RANS Reynolds Averaged Navier Stokes

SF Scale Factor

URANS Unsteady Reynolds Averaged Navier Stokes

A Area of solid/fluid heat transfer surface

C Specific heat capacity

$h$ Heat Transfer Coefficient

$k_{s} \quad$ Solid thermal conductivity

$L \quad$ Characteristic length

$m_{s} \quad$ Solid mass

$\mu$ Dynamic viscosity

$q_{w} \quad$ Wall heat flux

$\rho_{s} \quad$ Solid density

$R e_{a x}=\frac{\rho V_{a x} L}{\mu} \quad$ Reynolds number based on the flow axial velocity $V_{a x}$

$R e_{u}=\frac{\rho U L}{\mu} \quad$ Reynolds number based on the blade peripheral speed $U$

$R e_{u} \quad$ Reynolds number based on the rotor velocity

$t$ time

$T_{w} \quad$ Wall temperature

$T_{0} \quad$ Free-stream total inlet temperature

$\nabla T$ Temperature gradient

\section{Introduction}

Accurate prediction of unsteady thermal fields is a fundamental requirement in many engineering applications involving fluid-solid interaction-ranging from nuclear safety to chemical processing, electronics cooling, and power generation-being it for performance or safety concerns. Of particular interest for this contribution is the prediction of unsteady loads in the warm-up/cool-down phases of modern 
steam turbines: a problem becoming more and more prominent as the requirements for turbines' flexible operation increase. It is, however, in the authors' belief, that the discussion and conclusions are more general and far reaching and pertain to the general issue of unsteady Conjugate Heat Transfer (CHT) calculations.

Unsteady temperature evolution in a solid may be obtained through imposing a convective boundary condition at the fluid-solid interface-hence disregarding the fluid domain entirely. This approach has been recently applied by Topel et al. [1], [2], [3] in the context of steam turbine transient operation. While this approach can be successfully applied in some particular cases, it is far from having general validity. The assumption of Heat Transfer Coefficient (HTC) and driving temperature being independent of the wall thermal boundary condition, has been shown to be questionable, and a source of a long-standing debate in the academic community, as highlighted by Moffat [4].

Recent efforts by Marinescu et al. [5], considered removing the need to specify a convective boundary condition at the fluid-solid interface by including the fluid domain as a solid domain with a modified thermal conductivity. This over-conductivity approach shifts the issue from the determination of a consistent HTC to the empirical tuning of the over-conductivity value.

Given the shortcomings of the HTC and driving temperature as heat transfer descriptors, and the inherent empiricism of over-conductivity approaches, it becomes clear that a time-accurate unsteady CHT solution should be sought when high accuracy is required. This is particularly true for flow conditions where the aerodynamic and thermal field are strongly coupled [6], [7], [8].

A commonly adopted strategy for unsteady CHT is based on the quasi-steady assumption [9], [10], where the fluid domain is treated as stationary. This approach may introduce considerable errors in cases where the fluid domain presents unsteady time scales that are of the same order of the solid domain, as shown by He and Fadl [11].

The need for approximate strategies for computational modelling of unsteady CHT stems from the considerable time scale disparity between the solid and the fluid domains [12]. Time-marching of the conjugate solution in a monolithic solver, requires the time-stepping to be bound by the stability/accuracy of the fluid domain solution, with a dramatic impact on the computational cost and accuracy due to the accumulation of round-off errors in the solid domain. The issue of time scale disparity, which is due to the very physical nature of the problem, is a fundamental bottleneck in the application of unsteady CHT to problems of industrial relevance.

Amon [13] proposed to solve both the fluid and solid domain using a block spectral solver. This approach, however, did not solve the issue of the time scale disparity as both domains were still integrated using a time-marching approach.

In order to tackle this time-scale disparity in the case of periodic temperature disturbances in the solid, $\mathrm{He}$ and Oldfield [12] have examined the feasibility of treating the fluid and solid differently, in the time and frequency domains respectively. Expressing the temperature field in Fourier terms, and balancing the harmonic coefficients, it is possible to show [12] how the solution to the time-dependent energy equation can be obtained through two steady state solutions for the unknown harmonic coefficients. This allows to remove the constraint on time-marching both the solid and fluid domains using the same time-step as the solid solution is effectively obtained through steady calculation in the frequency domain. Recently He [14] extended the framework to a closely-coupled CHT method with Large Eddy Simulations (LES), where again, the solid domain is solved in a steady manner, and the unsteady conduction effect of the solid domain is included by a semi-analytical harmonic transfer function at the interface. These approaches suffer from the disadvantage of needing a Fourier solver for the solid domain, as well as an ad-hoc treatment of the fluid-solid interface allowing to account for the time/frequency transformation of boundary condition on the two sides of the solid/fluid interface. For this reason they have not seen yet a wide application.

For most practical situations, Reynolds Averaged Navier Stokes RANS have been and will continue to be the main flow modelling fidelity to be adopted. In the context of Unsteady Reynolds Averaged Navier Stokes (URANS) solutions, the following two approaches in particular have enjoyed a fair popularity for the unsteady CHT study of the flexible operation of steam turbines.

The first is the Multi-Scale Modelling (MSM) method (described in more detail inSec. 2.2), proposed by $\mathrm{He}$ and Fadl [11]. This approach is based on a dual time-scale formulation of the Navier-Stokes and Energy equations. Through manipulation of the Navier-Stokes and solid conduction equations, as well as appropriate treatment of the solid-fluid interface boundary condition, the method allows to march in time the solid solution with a time-step that is not bound by the fluid domain. The method has been shown to allow considerable speed-up with respect to a monolithic fully-coupled (FC) solution-where the same solver is used for both Navier-Stokes and solid conduction, with energy conservation at the fluid-solid interface being implicitly satisfied-without loss of accuracy ( [15], [16], [17], [18]).

The second approach is the Equalized Time-scale (ET) method [19], [20], [21], [22], [23]. It is based on balancing the solid and fluid domain's time scales by scaling the specific heat capacity of the solid. The method has been applied to quasi-steady aero-thermal analysis ( [19], [22], [23]), as well as to study the transient temperature evolution in and radial turbines [20] and steam turbines during pre-warming phase [21], with a particular regard to the thermal stress prediction of rotor wheels [20].

Both the ET and MSM approaches can be used in an existing solver. For this reason they have been enjoying considerable popularity in the last years. The ET method, in particular, offers the advantage of immediacy, needing no modifications at all with respect to an existing CHT solver. On the contrary, a detailed investigation of the limitations of such an approach-particularly with reference to the effect of the scaling of the specific heat capacity on the unsteady 
CHT effects-have not been fully addressed. Limited experimental evidence is available to support its validity and a direct comparison with a reference monolithic FC method has not been reported in open literatures to the present authors' knowledge.

Given the relevance of the issue of the prediction of unsteady thermal loads for a broad range of engineering applications, as well as the computational saving granted by the use of an ET method, the question of interest is its accuracy and general validity. This issue is the subject of the present work. The analysis will be focused on a particular test case: the pre-warming of a steam turbine stage-where hot air is used to gradually increase the temperature of the turbine to reduce the start-up time. It is however believed that the present observations, should be of more general and far-reaching relevance. The more specific questions to be addressed in this work are:

1. What are, if any, the sources of general uncertainty introduced by the alteration of the solid specific heat capacity in the ET method?

2. what is the difference in accuracy between the ET and MSM method for a typical unsteady flow in a prewarming phase of a steam turbine?

The two methods, together with the baseline method for the Fully-Coupled (FC) reference solutions, will be presented briefly in the next section. This is followed by the analysis and discussion of the potential differences of the two approaches through a simple case of unsteady temperature evolution in a warming solid body under forced convection. Finally the steam turbine pre-warming case will be introduced and the numerical sensitivities discussed. The comparisons in accuracy between the two methods of interest will be made against the baseline reference FC monolithic solution.

\section{Unsteady CHT Methods}

In the following sections the three time integration strategies used in this paper will be introduced. The reference FC solution strategy is addressed first (Sec. 2.1), followed by the MSM (Sec. 2.2) and the ET (Sec. 2.3) method.

\subsection{Fully-Coupled unsteady solution (FC)}

In a FC integration of the unsteady CHT solution, both the fluid and solid domains are solved with a monolithic approach. This means that the same solver and discretization method used for the fluid domain, is also used for the time integration of the solid solution. The same energy equation solved for the fluid domain is solved in the solid, of course considering different material properties and neglecting all the convective terms. In this way the fluid energy equation reduces to the Fourier conduction equation. The solid and fluid domains are part of the same computational grid, without the need to treat separately the fluid-solid interface. This

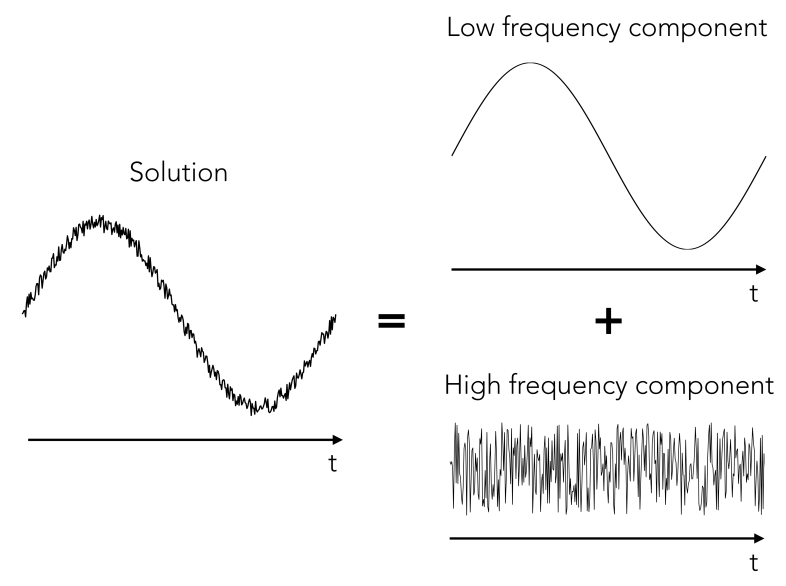

Fig. 1. Splitting of the low and high frequency scales of the solution in MSM method

automatically allows the conservation of energy and continuity of temperature at the CHT wall at each time-step.

Since both domains are marched in time using the same physical time-step, dictated by the fluid domain, this approach requires a considerable computational effort.

A FC solution, on the other hand, is not based on any simplified assumptions and can capture mutual interaction between the fluid and solid domain unsteady phenomena across the whole range of time scales of interest. For this reason it has been used as a reference for the comparison between MSM and ET methods.

ANSYS Fluent pressure-based solver with second order implicit time discretization has been used for the FC unsteady solution of the cases presented in Sec. 4 .

\subsection{Multi-scale Modelling (MSM)}

The MSM method has been first described by $\mathrm{He}$ and Fadl [11]. At the core of the method is the separation of the unsteady solution in the fluid domain $U$ in a low frequency $\bar{U}$ and a high frequency $U^{\prime}$ component (see Fig. 1).

$$
U(\vec{x}, t)=\bar{U}(\vec{x}, t)+U^{\prime}(\vec{x}, t)
$$

where the $\bar{U}$ component is obtained through a suitable lowpass filtering operation.

Given the broad difference in time scale sensitivity between the solid and the fluid domains, the method considers the solid to be insensitive to the high frequency fluctuations. In this respect then, the method considers the influence of the low frequency variations on the high frequency components of the solution but neglects the converse. This assumption, that has shown to have no impact on the accuracy of the solution ( [11], [15], [16], [17], [18]) holds the key to the computational savings allowed by the method. Governing equations 


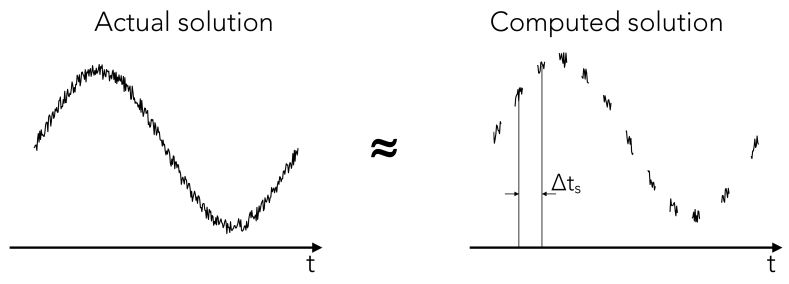

Fig. 2. Example of a computed solution in the MSM approach

in the fluid domain can be written in compact form as:

$$
\frac{\partial U}{\partial t}+R(U)=0
$$

Substituting the time-split formulation of Eq. (1) into Eq. (2) and applying low-pass filtering gives:

$$
\frac{\partial \bar{U}}{\partial t}+\overline{R(U)}=0
$$

It is possible to show [11] that Eq. (3) is equivalent to:

$$
\frac{\partial U}{\partial t}+R(U)=-\left.\frac{\partial \bar{U}}{\partial t}\right|_{S}
$$

where the source term on the right-hand side is the time derivative of the low-pass filtered solution calculated at a solid time-step as explained below.

The discretization of the two time derivatives in Eq. (4) is performed with two different resolutions. The term $\frac{\partial U}{\partial t}$ is sampled in order to capture the high frequency flow features of interest. This high frequency time-step of the fluid solution will be referred to as the fluid time-step $\left(\Delta t_{f}\right)$ in rest of the paper. On the contrary, being the low-pass filtered solution by definition invariant on the high frequency scales, sampling of the $\frac{\partial \bar{U}}{\partial t}$ term is only done at time intervals proper of the solid domain solution: the solid time-step $\left(\Delta t_{s}\right)$. The same value of $\Delta t_{s}$ is used for the time-marching of the solid domain. The names fluid time-step and solid time-step have been chosen as the first is used to resolve the high frequency flow features in the fluid domain and the latter to march in time the solid solution. However, while it is true that the solid solution is obtained with a time resolution of $\Delta t_{s}$, the fluid solution uses a multi-scale time integration on both time scales $\Delta t_{s}$ and $\Delta t_{f}$.

The use of a dual time-scale formulation for the fluid domain is a fundamental aspect of the advantage in computational time given by the MSM approach, compared to a FC solution. While a monolithic FC solution needs to sample the whole physical time using the fluid time step, a MSM approach allows a bigger time-step $\Delta t_{s}$ to reconstruct the lowfrequency scales as shown in Fig. 2.

Loosely-coupled communication of wall heat flux and temperature at the fluid-solid interface is enforced for each
$\Delta t_{s}$ until continuity of temperature and heat flux is satisfied. A detailed discussion of the work-flow of the solution strategy is shown in the original publication describing the method [11].

The MSM solver used for the results of Sec. 4 is based on the standard ANSYS Fluent pressure-based solver extended through User Defined Functions (UDF). The time discretization of the RHS in Eq. (4) is performed using a second order formulation for uneven time steps proposed by Maffulli et al. [18].

\subsection{Equalizing Time-scales (ET)}

The ET method has been first proposed by Diefenthal et al. [20]. At its core is a fully coupled monolithic CHT solver. The fundamental difference with the baseline FC solver described in Sec. 2.1, is in the material properties used in the solid domain. In the ET approach, the two different time scales of advective phenomena in the fluid domain and diffusive ones in the solid domain, are equalized through artificially altering the specific heat capacity of the solid.

Scaling down the specific heat capacity of the solid $C$ by a Scale Factor (SF) (Eqn. (5)) causes a parallel SF-fold decrease in the time constant of the solid domain's response. The ET method effectively uses a material with a reduced specific heat capacity $C_{E T}$.

$$
C_{E T}=\frac{C}{S F}
$$

If one disregards the effect of wall temperature on HTC, and more broadly the mutual interaction between heat transfer and aerodynamics, this operation does not alter the Fourier and Biot numbers [23], which should presumably be able to produce the same solutions under the same nondimensional parameters.

It is, however, questionable the level to which this approximation can be held valid in a typical turbomachinery unsteady compressible flow, where the aerodynamic and thermal field are strongly coupled. It is then of interest to understand the limitations of the ET method, if any, with respect to the MSM approach.

This will be addressed first (Sec. 3) through a simplified analysis of a heating body, followed by (Sec. 4) the unsteady analysis of a steam turbine pre-warming phase.

\section{Solid body subjected to unsteady convective heat flux}

Before considering a full-detailed CFD case, it is instructive to observe the differences between the two approaches through a simplified, but realistic, test case.

The test case considers the warming up of a solid body with uniform internal temperature, subjected to time-varying wall heat flux, under conditions that are representative of a pre-warming phase. The time evolution of the temperature- 


\begin{tabular}{ll} 
Density $(\rho)$ & $8030\left[\mathrm{~kg} / \mathrm{m}^{3}\right]$ \\
specific heat capacity $(C)$ & $502.48[\mathrm{~J} / \mathrm{kg}-\mathrm{K}]$ \\
Thermal conductivity $(k)$ & $16.27[\mathrm{~W} / \mathrm{m}-\mathrm{K}]$ \\
\hline
\end{tabular}

Table 1. Solid material properties used in both cases of Sec. 3 and Sec. 4

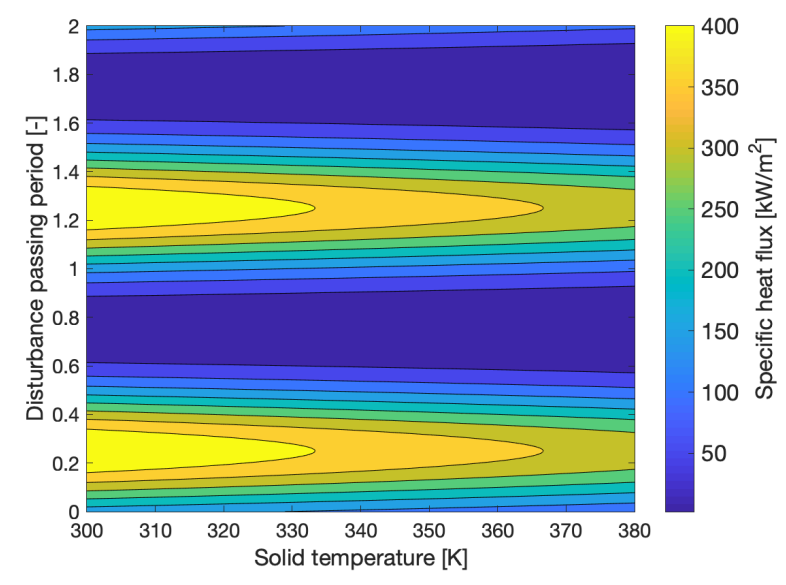

Fig. 3. Time and temperature dependence of the specific heat flux to the solid body test case. This is evaluated analytically considering that both $h(t)$ and $\left[T_{f}(t)-T(t)\right]$ vary as sine waves, as described in the text.

uniform in space—in the solid domain is dictated by:

$$
m C \frac{d T(t)}{d t}=A h(t)\left[T_{f}(t)-T(t)\right]
$$

where $m$ is the mass of the solid, $C$ its specific heat capacity, $A$ the surface area, $h(t)$ the time-varying HTC and $T_{f}(t)$ the time-varying driving temperature.

The solid has same volume, surface area and material properties (see Table 1) of the Intermediate Pressure (IP) turbine blade used in Sec. 4. Both HTC and driving temperature have a sinusoidal variation with the same frequency as the blade passing frequency of the rotor under pre-warming conditions. The HTC varies, in a period, between 500 and $50\left[\mathrm{~W} / \mathrm{m}^{2}-K\right]$. Driving temperature $T_{f}(t)$ varies between 400 and $350[K]$. These values are representative of a flow periodically passing through a wake generated by separated flow - as is the case for typical pre-warming conditions. The resulting periodic specific heat load to the solid body, as function of the solid temperature $T(t)$ is shown in Fig. 3.

Figure 4 shows the temperature evolution of the solid domain obtained using a direct integration, the multi-scale integration and an ET method using both a $10^{3}$ and $10^{4} \mathrm{SF}$, (defined in Eq. (5)). The time discretization for all methods uses a second order explicit time marching. Time steps used for the FC integration was $10^{-4}$ seconds. Multi-scale integration used the same value for the high frequency $\Delta t_{f}$. Filtering was carried out every 100 time-steps and the solid

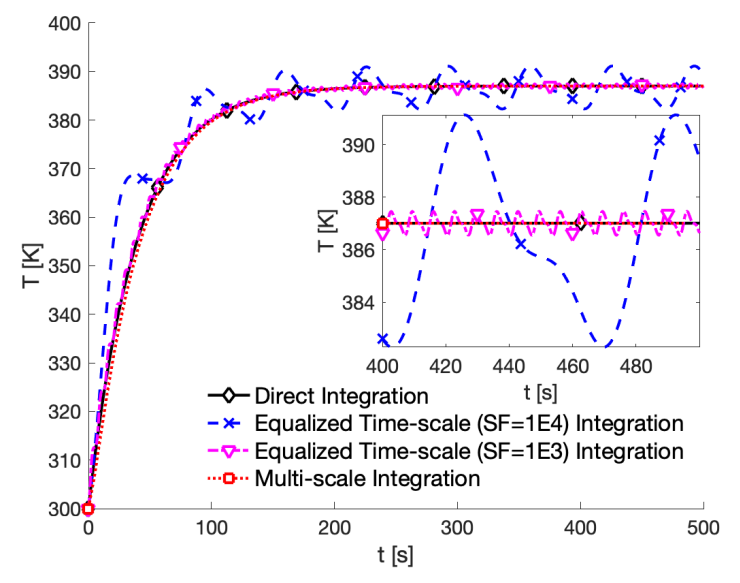

Fig. 4. Comparison of Direct, ET and MSM solutions for the solid body test case under heat load representative of pre-warming conditions

$\Delta t_{s}=5$ seconds. The ET solution uses a time-step that is equal to $10^{-4} / S F$ seconds. All results have been checked to be not dependent on the time discretization.

The results clearly show how the multi-scale method allows to reconstruct the time evolution of the temperature in the solid with a very close match to the direct integration. It should be noted that - although performance is not a concern for this simple case- the use of MSM allows for a 50-fold saving in terms of computational time with respect to a direct integration. The ET method has been applied to the present test case with two distinct scaling factors: $10^{4}$ and $10^{3}$. Both values have been used in the previous studies using the ET method, with $10^{4}$ been selected as reference for both quasisteady ( [23], [21]) and unsteady [20] calculations. It is evident, from Fig.4, that the ET method can capture, at least in a time-averaged sense, the steady state behaviour of the solid. There appears to be a threshold after which the effect of the increase in thermal sensitivity of the solid cannot be recovered after the linear scaling of the time axis. While the use of a SF of $10^{3}$ allows a reasonably accurate reconstruction of the unsteady solid temperature, a SF of $10^{4}$ results in a complete misprediction of the initial transient. Even in the steady response, the ET with SF of $10^{4}$ shows temperature fluctuations of about $10 \mathrm{~K}$, far from negligible when lifetime predictions are of interest. The use of a $10^{3} \mathrm{SF}$ allows for a better accuracy with respect to the baseline solution.

The sensitivity of the problem to the appropriate SF is naturally dictated by the ratio between heat load and heat capacity of the solid. It is then instructive to understand whether a SF of $10^{3}$ can be deemed appropriate also in the case of an increased thermal load. Figure 5 shows the temperature evolution in time in conditions that are representative of a warm start of a steam turbine. The solid body, initially at $400 \mathrm{~K}$, sees now a higher driving temperature (ranging from $850 \mathrm{~K}$ to $900 \mathrm{~K}$ ) as well as higher HTC (varying from 700 to $2000 \mathrm{~W} / \mathrm{m}^{2}-K$ ), representative of full load conditions. It appears that, for a high heat load case, also the use of a $10^{3} \mathrm{SF}$ seems inadequate to accurately predict 


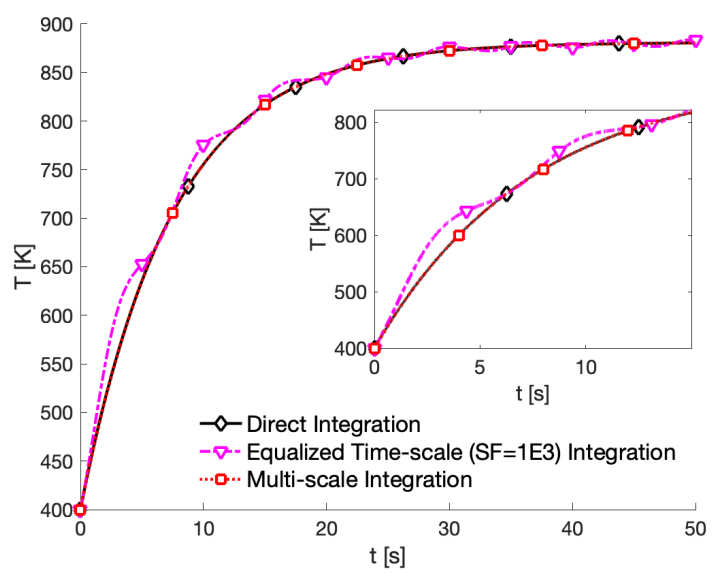

Fig. 5. Comparison of Direct, ET and MSM solutions for the solid body test case

the transient behaviour, with max temperature deviations in the order of $50 \mathrm{~K}$

The results shown in this section, though obtained using a simplified model, highlight a fundamental shortcoming of the ET method. The value of choice of the SF carries significant effects on both steady-state and transient thermal response. Furthermore, the choice of SF is heavily influenced by the ratio between the thermal load and the unscaled specific heat capacity of the solid domain, highlighting the empiricism necessary to fine-tune an ET model for a specific analysis. Given the results of this section, it is of interest to compare the performance predictions between the ET and MSM methods on a real test case of a pre-warming phase of a steam turbine. The analysis and results are presented in the following section.

\section{Unsteady CHT analysis of steam turbine pre- warming with hot air}

The progressive penetration of the renewable share in the energy market, has caused a profound change in the operation of traditional power plants. The growing request for more flexible energy generation of conventional steam turbines, has increased the necessity for rapid start-up/shutdown cycles. In this context, accurate prediction of thermal transients is critical with respect to Low Cycle Fatigue (LCF) life consumption [24]. Pre-warming of the machine with hot air can be used to effectively control the thermal stresses during the ramp-up phase [25], and has been chosen as a very relevant test case for a comparison of both MSM and ET methods.

\subsection{Case description}

The case considers a 1.5 stage IP turbine using realistic blade profiles. A 2D analysis has been deemed adequate for the purpose of the present work, without loss of generality.

The baseline solver used is the second order ANSYS Fluent pressure-based coupled solver. Time marching is performed using second order implicit time integration. The

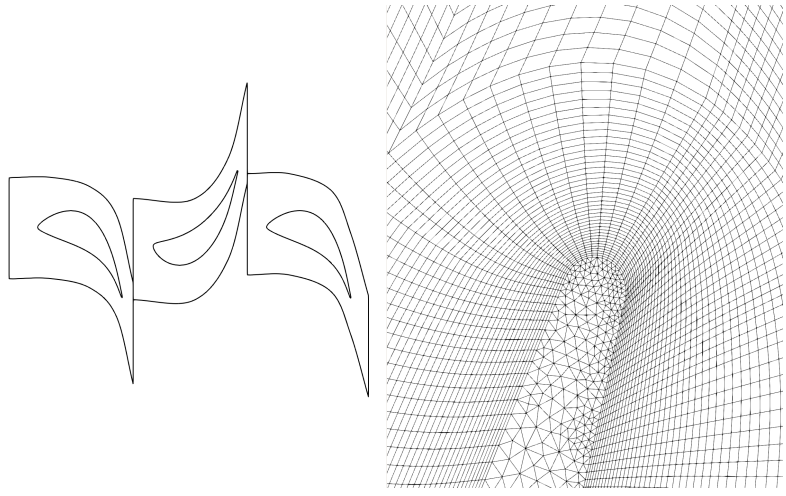

Fig. 6. Computational domain used with a close-up of the fluid-solid domain meshes for the rotor trailing edge region

MSM approach uses this time integration for the fluid timesteps (see Sec. 2.2), while for the solid time marching uses the second order method described by Maffulli et al. [18]. For the MSM and FC methods a sliding mesh calculation has been used. The performance of the ET method has been assessed considering both frozen rotor and sliding mesh as both have been applied in previous works using ET [22], [23]. No major differences between the two approaches have been observed and - for the sake of brevity - only the sliding mesh results are going to be shown in the following. Turbulence closure has been ensured using $k-\omega$ SST model. Wall refinement ensured $y^{+}<1$ on all the viscous walls. The computational domain used and details of the mesh are shown in Fig. 6.

The pre-warming phase has been modelled as a thermal shock process. Total inlet flow temperature is constant throughout the process and fixed at $400 \mathrm{~K}$. The solid is initially at uniform temperature of $300 \mathrm{~K}$. To set the fluid initial conditions, a first unsteady fluid-only calculation with constant $300 \mathrm{~K}$ wall temperature has been performed until time periodic flow conditions are established and initial flow transients have been convected out of the domain. The solid warming starts then at time 0 when the coupling between the solid and fluid domain is activated.

The mass flow rate and rotational speed of the rotor correspond to an axial Reynolds number $R e_{a x}=5500$ and a tangential Reynolds number $R e_{u}=6000$. The comparison between the FC, MSM and ET methods is established through the first 25 seconds of the transient, approximately $1 / 4$ of the whole warm-up time.

\subsection{Numerical sensitivities}

Two different grid densities have been considered: 320,000 and 600,000 nodes. Given the more strict requirements both in terms of space and time discretization, the grid and time sensitivity analysis has been performed on the fluid domain only. Three different time-steps have been considered: $1.6^{-4}, 1.0^{-4}$ and $6.5^{-5}$ seconds. These correspond respectively to 40,60 , and 100 time steps for blade passing period. The results of the grid and time step sensitivity analysis are summarized in Fig. 7. The figure shows the non- 


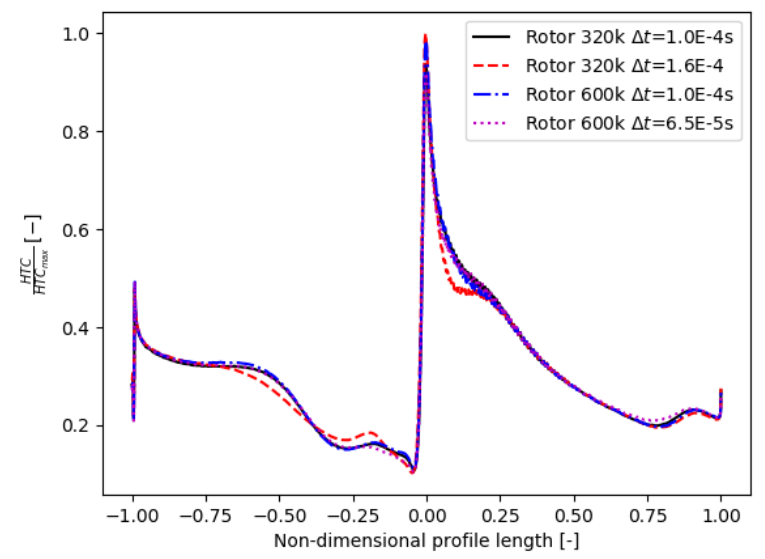

Fig. 7. Scaled HTC distribution on the rotor wall obtained through different grid and time step sizes. All profiles are scaled using a single reference value $H T C_{r e f}=\max \left(H T C_{F C}\right)$

dimensional HTC distributions on the rotor blade. The HTC has been defined throughout the paper as:

$$
H T C=\frac{q_{w}}{T_{0}-T_{w}}
$$

The results of Fig. 7 show that grid and time step independence has been reached using a mesh with 320,000 nodes and 60 time steps per blade passing period. This set-up has been used in the remaining part of the paper.

The results of Fig. 7 are averaged over 10 blade passages. This value has been obtained through a separate sensitivity analysis, the results of which are shown in Fig. 8, showing negligible effect of averaging after 10 blade passing periods. This value has been used also in the MSM for the filtering process, separating the solid and fluid scales.

\subsection{Unsteady CHT analysis of pre-warming operation}

The pre-warming simulation has been run for a physical time of 25 seconds, or equivalent in the case of ET cases. The results are summarized in this section.

Figure 9 shows the non-dimensional HTC distribution on the rotor surface at 15 seconds, as predicted by the different methods. The reference HTC used for scaling all distributions is the maximum HTC predicted by the FC simulation. Similarly to what was done for the filtering operation in the MSM, the FC simulation has been averaged across 10 blade passing periods. A similar averaging has not been possible due to the compression of the time axis for the ET results: in both cases the simulation was marched in time for less than 10 blade passages. It should be stressed, again, that all simulations started with the same initial conditions, where a fully developed flow was ensured and any initial transients vanished. A very good match between the FC and MSM HTC distributions can be observed. The ET simulation using a $\mathrm{SF}=10^{3}$ shows a reasonably close prediction to the $\mathrm{FC}$,

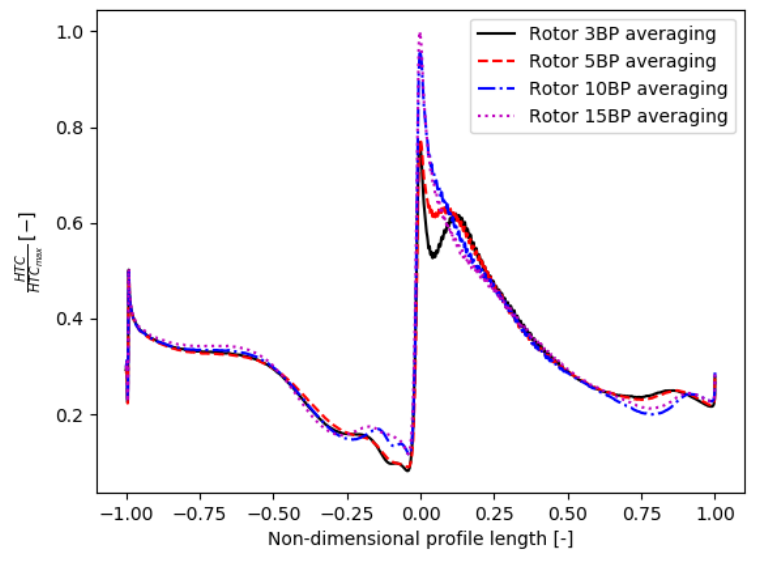

Fig. 8. Scaled HTC distribution on the rotor wall obtained through averaging over different number of blade passing periods. All profiles are scaled using a single reference value $H T C_{r e f}=$ $\max \left(H T C_{F C}\right)$

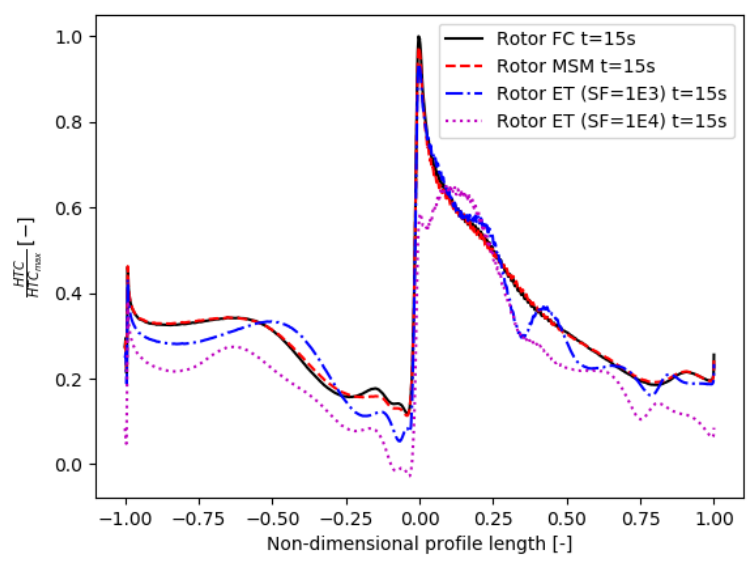

Fig. 9. Scaled HTC distribution on the rotor wall after 15 seconds: comparison of ET, MSM and FC method. All profiles are scaled using a single reference value $H T C_{r e f}=\max \left(H T C_{F C}\right)$.

while the one using $\mathrm{SF}=10^{4}$ considerably under-predicts the heat load on the profile. The average HTC predicted using ET method with a $\mathrm{SF}=10^{4}$ is $23 \%$ lower than the baseline FC method. Similar trends have been observed for the second stator, the results of which are not reported here for the sake of brevity.

The HTC predictions have a direct impact on the surface temperature field. Comparison of the non-dimensional wall temperature distribution on the rotor is shown in Fig. 10. Also in this case the temperature distributions for all the methods have been scaled with a the same reference temperature, equal to the maximum temperature predicted by the FC method. In accordance to what is suggested by the HTC results of Fig. 9, the FC, MSM, and ET method using $\mathrm{SF}=10^{3}$ show similar accuracy with respect to the wall temperature prediction. On the contrary the ET method us- 


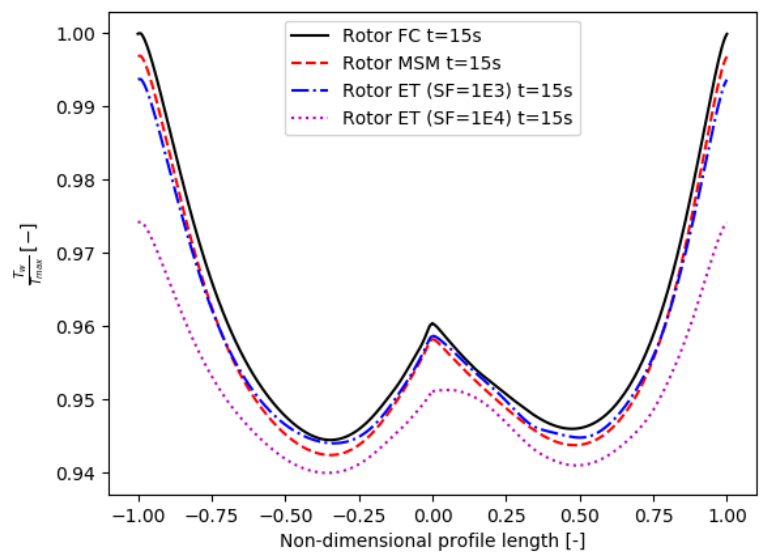

Fig. 10. Scaled temperature distribution on the rotor wall after 15 seconds: comparison of ET, MSM and FC method. All profiles are scaled using a single reference value $T_{r e f}=\max \left(T_{F C}\right)$.

ing $\mathrm{SF}=10^{4}$ consistently under-predicts the wall temperature on the whole rotor. The differences in predicted wall temperature between the FC method and the ET method using $\mathrm{SF}=10^{4}$ grow up to $3 \%$ at the trailing edge: a value that can have dramatic impact on the life prediction of the turbine. Also in this case, the results of the second stator have been observed to have similar trends.

Figure 11 shows the non-dimensional contours after 15 seconds in the solid domain predicted by the considered methods. The results obtained using ET using $\mathrm{SF}=10^{4}$ show that the whole domain is predicted to have a considerably lower temperature than the baseline FC method. Similar accuracy is achieved between the FC, MSM and ET method using $\mathrm{SF}=10^{3}$.

The results shown so far confirm, as expected, the discussion in Sec. 3. Using a $\mathrm{SF}=10^{4}$, given the considered case, seems to be not appropriate for the accurate prediction of the unsteady thermal load in the present case.

One aspect-crucial for the correct estimation of the thermal stresses, and prediction of life consumption of the machine under thermal transients-is the correct calculation of the thermal gradients in the solid. It is of interest, thus, to explore what the effect of scaling the solid specific heat capacity on the distribution of $\nabla T$ is. Figure 12 shows distribution of the magnitude of the temperature gradient vector for $\mathrm{t}=15 \mathrm{~s}$ predicted using the three different methods considered in this paper. Similarly to what was done previously, all the distributions shown are scaled using a single constant reference value for the magnitude of $\nabla T$ : the maximum value of $|\nabla T|$ predicted by the baseline FC solver.

The results of Fig. 12 show a very good agreement between the baseline FC solution and the MSM one. In a clear contrast on the other hand, scaling the solid specific heat capacity in the ET method has considerable influence on the thermal gradient predictions. This is true also for the case with $\mathrm{SF}=10^{3}$-for which the temperature predictions were overall acceptable - particularly in the region of the trailing

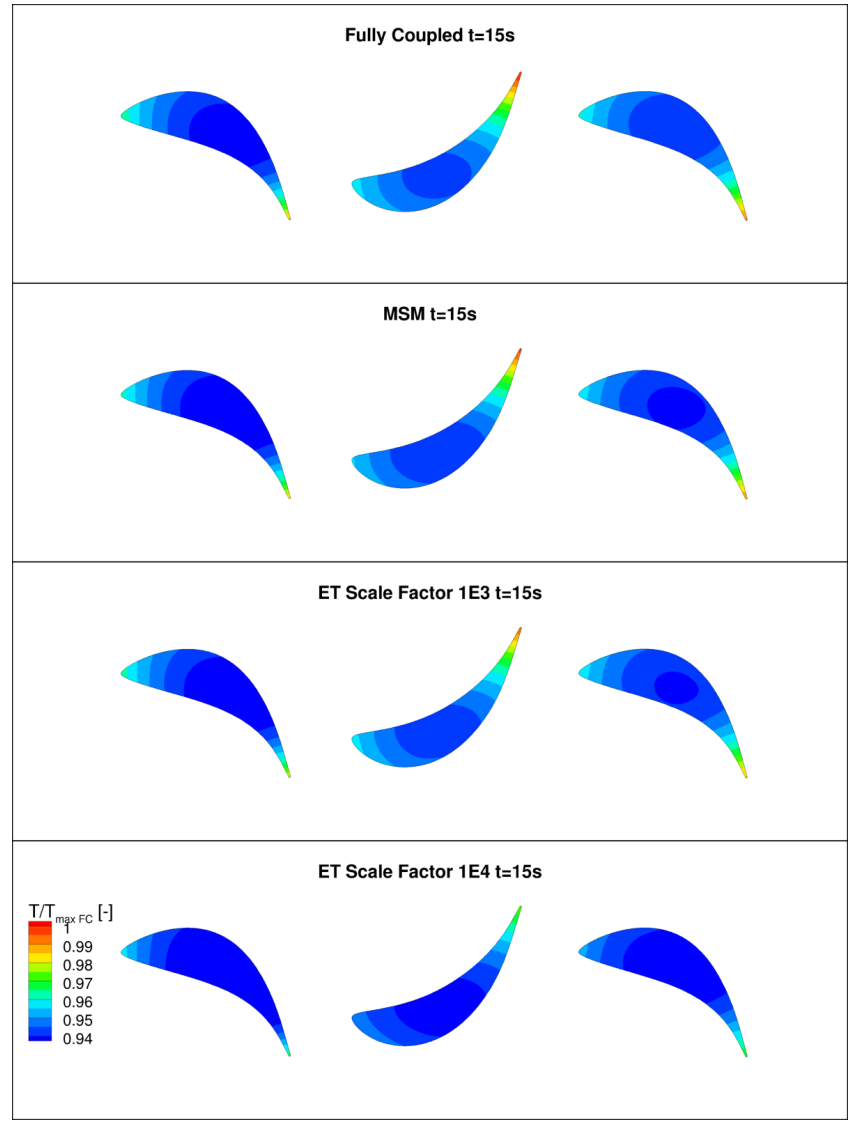

Fig. 11. Scaled temperature field in the solid domain after $15 \mathrm{sec}-$ onds: comparison of ET, MSM and FC method. All profiles are scaled using a single reference value $T_{\text {ref }}=\max \left(T_{F C}\right)$.

edge of both the rotor and second stator. The use of a $\mathrm{SF}=10^{4}$ causes an overall reduction of the values of the thermal gradient with respect to the baseline solution-as expected given the reduced effective thermal capacity of the solid — as well as a general mismatch of the iso-contours given the different penetration lengths of the unsteady disturbances enabled by the reduced specific heat capacity.

Given the importance of correctly predicting the maximum temperature gradient for accurate life estimation prediction, a more detailed analysis regarding the evolution of the maximum $\nabla T$ in the solid domain has been performed. The results are reported in Fig. 13. The figure shows the evolution in time of the non-dimensional maximum value of the temperature gradient in the rotor predicted by the three methods. The values have been non-dimensionalized using a single constant reference value equal to the maximum $|\nabla T|$ predicted by the baseline FC solver at $\mathrm{t}=0 \mathrm{~s}$. The superior accuracy offered by the MSM model, with respect to the ET method, when the thermal gradients are of interest is clear. The ET method using $\mathrm{SF}=10^{3}$ underpredicts the thermal gradient by up to $20 \%$ in the initial phases of the transient. An increase of the SF to $10^{4}$ causes discrepancies to rise up to $50 \%$. Both analysed scaling factors for the ET method revealed to be considerably inadequate when an accurate prediction of thermal gradients is of interest. Similar results are 


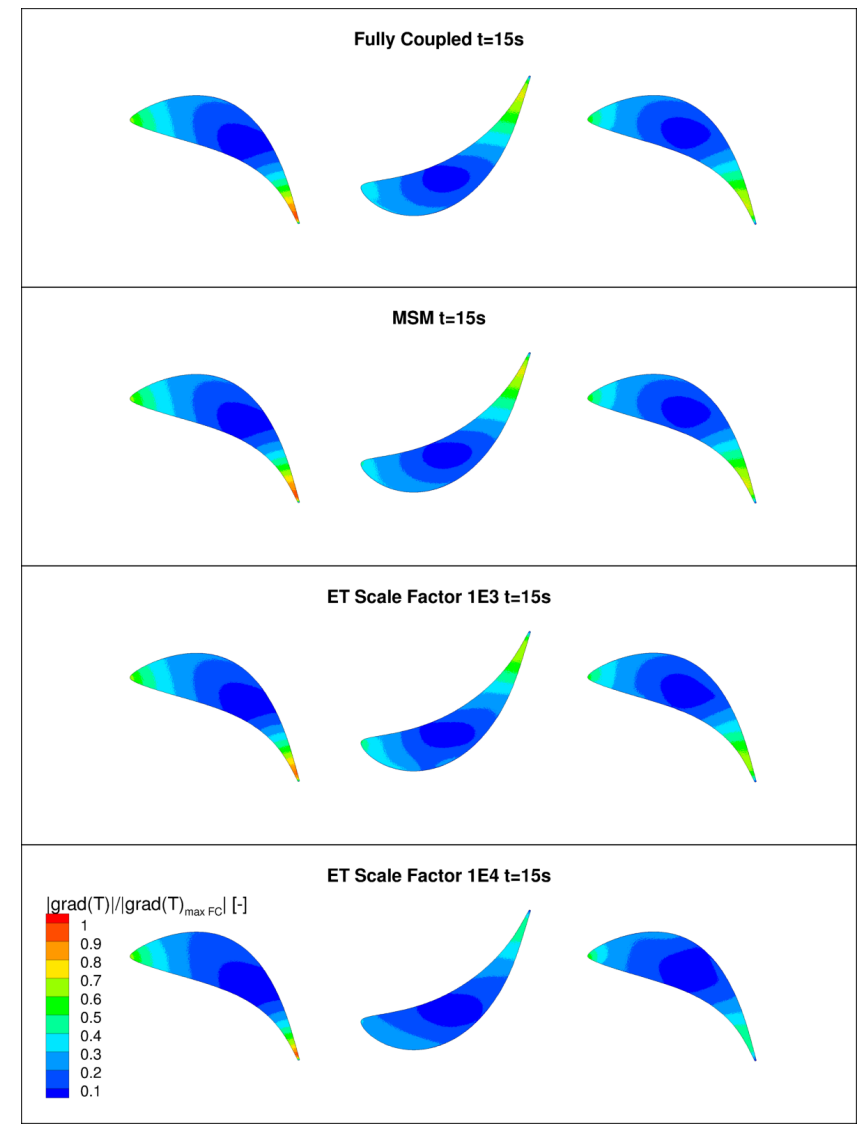

Fig. 12. Scaled temperature gradient field in the solid domain after 15 seconds: comparison of ET, MSM and FC method. All values are scaled using a single reference value $\left|\nabla T_{r e f}\right|=\max \left(\left|\nabla T_{F C}\right|\right)$.

observed for the second stator.

As for the computational cost of the considered methods it should be mentioned that the MSM achieved - as shown in this section - a very good accuracy with respect to the baseline FC model with a 50-fold speed-up. The two ET methods had a speed-up equal to the used scale factor but showed nonnegligible issues with their accuracy.

The results shown and discussed above consider onlyfor the sake of brevity-ET simulations obtained using a sliding mesh rotor-stator interface. The authors investigated also the use of a frozen rotor interface (applied in several publications adopting the ET method e.g. [22], [23]). Both sliding mesh and frozen rotor results lead to the same conclusions regarding the applicability of the ET method for unsteady thermal load prediction.

\section{Concluding remarks}

It is of interest to understand the relative advantages and drawbacks of two recently developed methods, the MultiScale Model (MSM) and Equalized Time-scale (ET) for efficient and accurate transient Conjugate Heat Transfer, in comparison with a baseline fully coupled (FC) solution commonly available. This has not been previously documented and it is the main contribution of the present work.

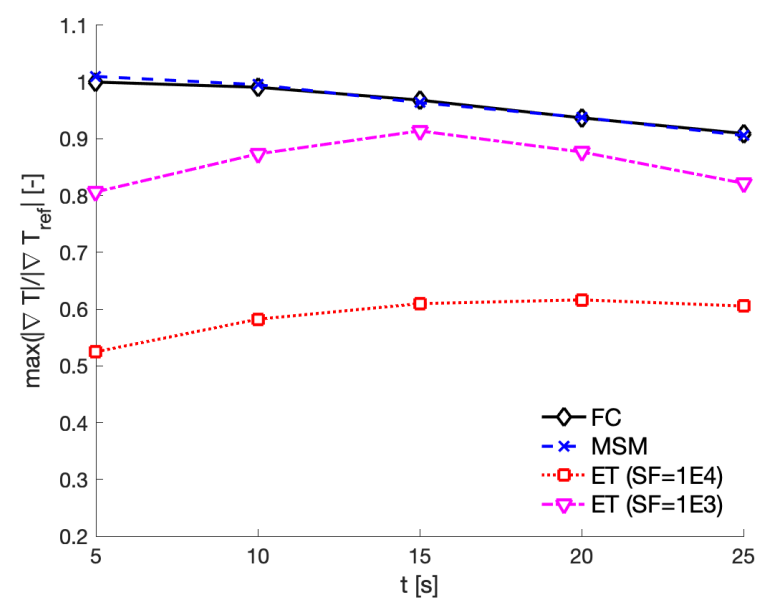

Fig. 13. Evolution of the maximum scaled temperature gradient with time: comparison of ET, MSM and FC method. All values are scaled using a single reference value $\left|\nabla T_{\text {ref }}\right|=\max \left(\left|\nabla T_{F C}\right|\right)$.

A first analysis has been conducted on a simple test case of a solid body with uniform temperature subjected to a variable thermal load (Sec. 3). The results have shown that the accuracy of the ET method strongly depends on the ratio between the specific heat capacity of the solid and the external thermal load. This introduces a considerable source of uncertainty regarding the results of an ET analysis, that in most cases cannot be accurately supported due to the absence of corresponding FC simulations. On the contrary, the MSM approach showed similar accuracy to the baseline solution for both thermal load conditions analysed.

A more realistic case of pre-warming of a steam turbine has been discussed in Sec. 4. The case considers the temperature evolution in the rotor and blade rows during the pre-warming phase. The MSM approach has shown a similar accuracy with respect to the baseline FC method, with a speed up factor of 50 in terms of computational time. The ET method using a SF of $10^{3}$ has shown good accuracy for what concerns the wall temperature and HTC predictions. An increase of the SF to $10^{4}$ - a value that has been reported in literature (e.g. [23], [22])—causes a non-negligible underestimation of both the wall temperature and heat load.

When considering also the magnitude of the thermal gradients in the solid domain, however, the ET method has revealed considerable shortcomings when using both the considered scale factors. The decreased solid specific heat capacity causes the ET method to under-predict the thermal gradients. Particularly the ET method with a $\mathrm{SF}=10^{3}$ predicted the maximum $|\nabla T|$ in the rotor to be $80 \%$ of the baseline value. The use of a $\mathrm{SF}=10^{4}$ resulted in a maximum $|\nabla T|$ equal to $50 \%$ of what was predicted by the baseline FC simulation. The MSM model has, however, shown very good accuracy with respect to the baseline solution.

This, three main conclusions can be drawn from the present work:

1. the accuracy of the ET method strongly depends on the choice of the SF; 
2. optimal value of the SF for the ET method depends, in turn, on the external heat load, adding to the empiricism in tuning the method-particularly with reference to a realistic 3D geometry where thermal loads may be markedly different in different flow regions-and to its general uncertainty in absence of strong validation datasets;

3. the increase of the specific heat capacity in the ET method causes a non negligible (up to $50 \%$ in the present case of a turbine blading) underestimation of the thermal gradients in the solid;

4. the MSM approach has been shown to offer a robust and accurate solution strategy, with a considerable (50-fold for the case of Sec. 4) reduction in computational time with respect to the baseline $\mathrm{FC}$ solution at no loss of accuracy.

\section{References}

[1] Topel, M., Nilsson, A., Jöcker, M., Spelling, J., and Laumert, B., 2018. "Investigation into the thermal limitations of steam turbines during start-up operation". Journal of Engineering for Gas Turbines and Power, 130(1), p. 012603.

[2] Topel, M., Genrup, M., Jöcker, M., Spelling, J., and Laumert, B., 2015. "Operational improvements for startup time reduction in solar steam turbines". Journal of Engineering for Gas Turbines and Power, 137(4), p. 042604.

[3] Topel, M., Jöcker, M., Paul, S., and Laumert, B., 2016. "Differential expansion sensitivity studies during steam turbine startup". Journal of Engineering for Gas Turbines and Power, 138(6), p. 062102.

[4] Moffat, R. J., 1998. "What's new in convective heat transfer?". International Journal of Heat and Fluid Flow, 19(2), pp. 90-101.

[5] Marinescu, G., Mohr, W. F., Ehrsam, A., Ruffino, P., and Sell, M., 2014. "Experimental investigation into thermal behavior of steam turbine components-temperature measurements with optical probes and natural cooling analysis". Journal of Engineering for Gas Turbines and Power, 136(2), p. 021602.

[6] Maffulli, R., and He, L., 2014. "Wall temperature effects on heat transfer coefficient for high-pressure turbines". Journal of Propulsion and Power, 30(4), pp. 1080-1090.

[7] Maffulli, R., and He, L., 2017. "Impact of wall temperature on heat transfer coefficient and aerodynamics for three-dimensional turbine blade passage". Journal of Thermal Science and Engineering Applications, 9(4), p. 041002.

[8] Jiang, H., Zhang, Q., He, L., Lu, S., Wang, L., and Teng, J., 2018. "Experimental evidence of temperature ratio effect on turbine blade tip heat transfer". Journal of Turbomachinery, 140(12), p. 121010.
[9] Sun, Z., Chew, J. W., Hills, N. J., Volkov, K. N., and Barnes, C. J., 2010. "Efficient finite element analysis/computational fluid dynamics thermal coupling for engineering applications". Journal of turbomachinery, 132(3), p. 031016.

[10] Wang, Z., Corral, R., Chaquet, J. M., and Pastor, G., 2013. "Analysis and improvement of a loosely coupled fluid-solid heat transfer method". In ASME Turbo Expo 2013: Turbine Technical Conference and Exposition, American Society of Mechanical Engineers, pp. V03BT11A006-V03BT11A006.

[11] He, L., and Fadl, M., 2017. "Multi-scale time integration for transient conjugate heat transfer". International Journal for Numerical Methods in Fluids, 83(12), pp. 887-904.

[12] He, L., and Oldfield, M., 2011. "Unsteady conjugate heat transfer modeling". Journal of turbomachinery, 133(3), p. 031022.

[13] Amon, C. H., 1995. "Spectral element-fourier method for unsteady conjugate heat transfer in complex geometry flows". Journal of thermophysics and heat transfer, 9(2), pp. 247-253.

[14] He, L., 2019. "Closely coupled fluid-solid interface method with moving-average for les based conjugate heat transfer solution". International Journal of Heat and Fluid Flow, 79, p. 108440.

[15] Fadl, M., He, L., Stein, P., and Marinescu, G., 2018. "Assessment of unsteadiness modeling for transient natural convection". Journal of Engineering for Gas Turbines and Power, 140(1), p. 012605.

[16] Fadl, M., Stein, P., and He, L., 2018. "Full conjugate heat transfer modelling for steam turbines in transient operations". International Journal of Thermal Sciences, 124, pp. 240-250.

[17] Fadl, M., and He, L., 2017. "On les based conjugate heat transfer procedure for transient natural convection". In ASME Turbo Expo 2017: Turbomachinery Technical Conference and Exposition, American Society of Mechanical Engineers, pp. V05AT10A002V05AT10A002.

[18] Maffulli, R., He, L., Stein, P., and Marinescu, G., 2018. "Fast conjugate heat transfer simulation of long transient flexible operations using adaptive time stepping". Journal of Turbomachinery, 140(9), p. 091005.

[19] Toebben, D., Łuczyński, P., Diefenthal, M., Wirsum, M., Reitschmidt, S., Mohr, W. F., and Helbig, K., 2017. "Numerical investigation of the heat transfer and flow phenomena in an ip steam turbine in warmkeeping operation with hot air". In ASME Turbo Expo 2017: Turbomachinery Technical Conference and Exposition, American Society of Mechanical Engineers, pp. V008T29A014-V008T29A014.

[20] Diefenthal, M., Łuczyński, P., Rakut, C., Wirsum, M., and Heuer, T., 2017. "Thermomechanical analysis of transient temperatures in a radial turbine wheel". Journal of Turbomachinery, 139(9), p. 091001.

[21] Łuczyński, P., Erdmann, D., Többen, D., Wirsum, M., Helbig, K., and Mohr, W., 2018. "Fast numerical calcu- 
lation approaches for the modelling of transient temperature fields in a steam turbine in pre-warming operation using hot air". In GPPS Forum, pp. 10-12.

[22] Łuczyński, P., Többen, D., Wirsum, M., Mohr, W. F., and Helbig, K., 2017. "Modeling of warm-keeping process with hot air in steam turbines". Journal of Power Technologies, 97(5), p. 416.

[23] Łuczyński, P., Toebben, D., Wirsum, M., Mohr, W. F., and Helbig, K., 2019. "Unsteady conjugate heat transfer investigation of a multistage steam turbine in warmkeeping operation with hot air". Journal of Engineering for Gas Turbines and Power, 141(1), p. 011005.

[24] Born, D., Stein, P., Marinescu, G., Koch, S., and Schumacher, D., 2017. "Thermal modeling of an intermediate pressure steam turbine by means of conjugate heat transfer-simulation and validation". Journal of Engineering for Gas Turbines and Power, 139(3), p. 031903.

[25] Marinescu, G., Sell, M., Ehrsam, A., and Brunner, P. B., 2013. "Experimental investigation into thermal behavior of steam turbine components: Part 3-startup and the impact on lcf life". In ASME Turbo Expo 2013: Turbine Technical Conference and Exposition, American Society of Mechanical Engineers, pp. V03CT14A006-V03CT14A006. 